\title{
Simultaneous refractive index sensing using an array of suspended porous silicon membranes
}

\author{
David Martín-Sánchez, Todora Angelova and Jaime García-Rupérez, Senior Member, IEEE
}

\begin{abstract}
We propose a fast and cost-effective method for obtaining a miniaturized array-formatted sensor suitable for multiplexed detection. Our solution is based on the fabrication of multiple $\mu \mathrm{m}$-sized suspended porous silicon (PSi) membranes working as independent transducers. Our process can potentially integrate an array of up to 1000 sensing spots per $\mathrm{cm}^{2}$. We also propose a simple and user-friendly optical platform to simultaneously interrogate each element of the array in real-time. The feasibility of this idea was proved performing several sensing experiments where we were able to detect refractive index (RI) variations with different transducers at the same time. An average experimental sensitivity of $685 \mathrm{~nm} / \mathrm{RIU}$ (Refractive Index Unit) was achieved, with a theoretical limit of detection (LoD) of $10^{-5}$ RIU. The analyzed sensing spots displayed similar behavior both in time and in magnitude. We believe that the high capabilities of the sensor presented in this work, along with the sensing mechanism, can be very useful for multi-parametric analysis and multi-target detection of biological samples.
\end{abstract}

Index Terms - membrane, multiplexing, nanofabrication, photonic sensor, porous silicon.

\section{INTRODUCTION}

$\mathrm{B}$ IOSENSORS are low-cost, fast-response and easy-to-use analytical devices used for food safety, environmental control, healthcare and chemical and biological threats detection [1-3]. New materials are constantly proposed as transducing platforms to meet the ever-increasing demands for sensitivity or limit of detection (LoD) [4-7]. Porous silicon (PSi) is one of these, a microstructured material obtained after creating holes in a $\mathrm{Si}$ substrate, with an extremely broad internal surface suitable for the detection of low concentrations of target analytes [8].

PSi is mostly fabricated by anodization of a $\mathrm{Si}$ wafer [9]. This method consists on the partial dissolution of the Si crystal by a hydrofluoric acid (HF) based electrolyte while an electric field controls the free charges available during the reaction. Pore diameter and density can be tuned varying the current density, which in fact can be done continually during the etching process to obtain several layers with different porosities. Hence, photonic sensors such as Bragg reflectors [10] and microcavity structures [11] can be easily made with PSi, with high quality factor (Q-factor) spectral features. Anodization is much more

Submitted for review on November 7th, 2019. This work was supported by the Ministry of Economy and Competitiveness and Spanish National Research Council under project TEC2015-63838-C3-1-R-OPTONANOSENS and by the Generalitat Valencia under project AVANTI /2019/123. efficient in terms of cost and fabrication time, in comparison with other methods that create orderly porous materials by means of several lithographic processes [12,13].

The displacement of the spectral response of the PSi microstructures is linked to the refractive index (RI) variation of the medium filling the pores, whereas this is caused by the infiltration of gas, liquid or the adhesion of target analytes to the pore walls [14-16]. Previous studies have demonstrated a very low LoD of PSi-based sensors, down to $10^{-7}$ RIU [10,17], which has enabled the detection of biomolecules in a concentration range of interest for multiple biomedical applications [18,19]. It has also been proved that PSi-based RI sensors can reach sensitivities on the order of $10^{3} \mathrm{~nm} / \mathrm{RIU}$, much higher than the ones that can be obtained with non-porous sensors with a similar Q-factor [10, 20]. Besides, new techniques based on Tamm/Fano resonances promise to reach figures of merit (FOM) up to $10^{3} \mathrm{RIU}^{-1}$ with these structures [21].

Multiplexed detection of RI variations using multiple sensing spots enhances the reliability and robustness of the sensor, since the analysis of a population statistically improves the accuracy of the result [22-24]. Moreover, this configuration allows multitarget detection, which provides more relevant and concluding information concerning the composition of a complex sample [25,26]. PSi sensors can be patterned to obtain an array of sensing spots, whether chemically of physically. Several techniques have been proposed to confer multiple biological functionalities to the structure [8,27-30] but, in general, they require a complex fluidic system or a more elaborated biofunctionalization. On the other hand, physical patterning of PSi creates multiple transducers working independently, by locally anodizing the substrate or by splitting the already anodized area using lithography [31-33].

The expanding capabilities of these sensors require faster and more complex sensing platforms, able to address each sensing spot individually. Traditional optical setups consisting of an optical source and a spectrum analyzer are being replaced with digital cameras that record all signals simultaneously or scanners that interrogate each transducer sequentially [34-36]. Some other solutions include multiple wavelength interrogation or exploiting plasmonic and fluorescent properties [37,38]. All these new elements determine the acquisition time, which most

D. Martín-Sánchez, T. Angelova and J. García-Rupérez are with the Nanophotonics Technology Center (NTC), Universitat Politècnica de València, Valencia 46022, Spain (e-mail: jaigarru@ntc.upv.es). 
applications require to be minimum to allow real-time detection and continuous monitoring, and also the cost of the device.

In our work, we present a fast, cost-effective and easy-toimplement approach for obtaining multiple $\mu \mathrm{m}$-sized PSi sensing spots, consisting of a four-step fabrication process. First, we grew a $\mathrm{Si}$ oxide $\left(\mathrm{SiO}_{2}\right)$ layer on a $\mathrm{Si}$ substrate. Second, we patterned this layer using a manually shaped tape and etched it with HF vapor. Third, we used that pattern as a masking layer before wet etching with potassium hydroxide $(\mathrm{KOH})$ to locally reduce the thickness of the substrate. Fourth, we anodized the substrate to form an array of suspended PSi membranes in the wet etched regions. Our results prove the feasibility of this fourstep process for creating a 2D matrix of sensing elements with a width as small as $150 \mu \mathrm{m}$ and a thickness down to $20 \mu \mathrm{m}$. We believe that this method is a good alternative to traditional photolithographic techniques since it considerably reduces the fabrication time, the complexity of the process and its cost, while it adds some interesting functions such as local anodization of the substrate.

Additionally, we propose a simple optical system for fast alignment of the sensor, consisting of a tunable laser source and an infrared camera that acquired simultaneously and in realtime the transmittance spectra of all the sensing spots. The capabilities of the system were tested performing several RI sensing experiments, obtaining a sensitivity of $685 \mathrm{~nm} / \mathrm{RIU}$ (Refractive Index Unit), a FOM of $27.5 \mathrm{RIU}^{-1}$ and a theoretical LoD of $10^{-5}$ RIU. We believe that this system could be easily adapted in the future to use visible light sources and commercial cameras for smartphone applications.

\section{MAterials AND MethodS}

\section{A. Fabrication of the 2D matrix of PSi sensing spots}

Heavily doped p-type $\mathrm{Si}$ wafers (boron doped, <100> oriented, $0.016-0.018 \Omega \cdot \mathrm{cm}$ of resistivity, double side polished, $400-450 \mu \mathrm{m}$ of thickness) were purchased from MicroChemicals $\mathrm{GmbH}$ (Ulm, Germany). Sulfuric acid $\left(\mathrm{H}_{2} \mathrm{SO}_{4}\right)$, hydrogen peroxide $\left(\mathrm{H}_{2} \mathrm{O}_{2}\right)$ and hydrofluoric acid $(\mathrm{HF})$ (48 wt. \% in $\mathrm{H} 2 \mathrm{O}$ ) were purchased from BASF (Ludwigshafen am Rhein, Germany). Potassium hydroxide $(\mathrm{KOH})$ was obtained from Panreac S.L. (Castellar del Vallès, Spain). Acetone, ethanol (EtOH) and 2-Propanol (IPA) were purchased from Scharlab S.L. (Sentmenat, Spain). Deionized water (DIW) was produced in house by a Millipore water purification system.

First, the wafers were cut in pieces of $1.5 \times 1.5 \mathrm{~cm}^{2}$ and cleaned during $5 \mathrm{~min}$ in a $3: 1$ volumetric mixture of $\mathrm{H}_{2} \mathrm{SO}_{4}$ and $\mathrm{H}_{2} \mathrm{O}_{2}$, for removing organic residues off the surface. Then, the thickness of the substrate was reduced in several areas and PSi structures were formed on the back side, following the steps detailed below.

The first step of the fabrication process was the deposition of a $\mathrm{SiO}_{2}$ mask layer by Plasma Enhanced Chemical Vapour Deposition (PECVD), used to perform a selective wet etching of the substrate (Fig. 1a). The reaction was carried out at 400 ${ }^{\circ} \mathrm{C}$ and a pressure of $3.6 \mathrm{mbar}$, with a radiofrequency power set at $270 \mathrm{~W}$. Silane $\left(\mathrm{SiH}_{4}\right)$ and nitrous oxide $\left(\mathrm{N}_{2} \mathrm{O}\right)$ gases were

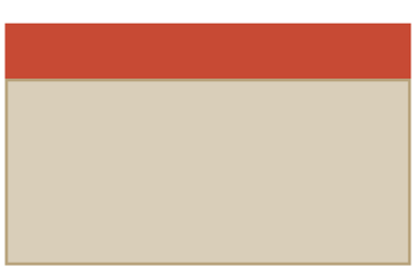

(a)

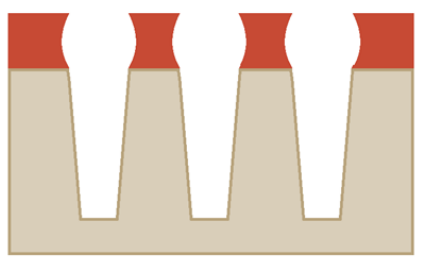

(c)

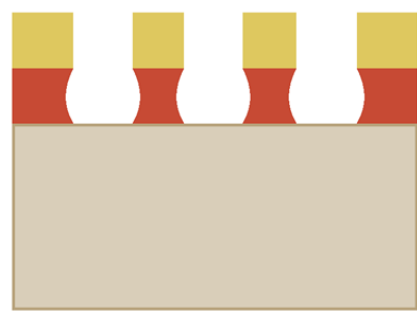

(b)

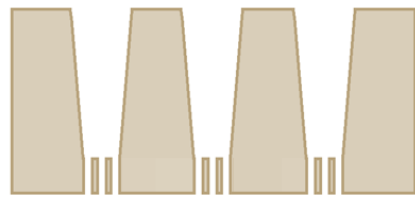

(d)

Notes:

Silicon substrate

Fig. 1. Four-step fabrication process for obtaining suspended PSi membranes by selectively removing the substrate. (a) Deposition of a silicon dioxide, $\mathrm{SiO}_{2}$, mask layer with a thickness of $3.55 \pm 0.05 \mu \mathrm{m}$ by PECVD; (b) HF vapor etching of the masking layer during 12 min using Kapton ${ }^{\circledR}$ tape to expose the $\mathrm{SiO}_{2}$ layer; (c) Anisotropic wet etching of the silicon substrate with a KOHbased solution at $80^{\circ} \mathrm{C}$ for 7 hours in order to reduce the thickness down to 20 $\mu \mathrm{m}$; (d) Porosification of the backside of the substrate with an electrochemical etching using a HF-based electrolyte in combination with an organic compound.

used to form $\mathrm{SiO}_{2}$, which was deposited over the surface during $264 \mathrm{~s}$. A uniform layer covering the top side of the $\mathrm{Si}$ substrate was achieved with a thickness of $3.55 \pm 0.05 \mu \mathrm{m}$. Next, the $\mathrm{SiO}_{2}$ layer was patterned using a Kapton ${ }^{\circledR}$ tape adhered by hand, which had been manually punctured previously in order to create holes of $1 \mathrm{~mm}$ of diameter. Subsequently, the $\mathrm{SiO}_{2}$ coated samples were exposed to HF vapor, obtained from a $48 \mathrm{wt}$ \% in $\mathrm{H} 2 \mathrm{O}$ solution at room temperature (Fig. 1b). The HF vapor etched the $\mathrm{SiO}_{2}$ isotropically at a rate of approximately $5 \mathrm{~nm} / \mathrm{s}$ and had no effect on the Kapton ${ }^{\circledR}$ film and neither on Si. The oxide layer was etched during $12 \mathrm{~min}$ in order to remove the whole thickness. At the end, each piece was rinsed with DIW and cleaned with acetone and IPA.

After the patterning of the surface, the exposed Si was deepetched anisotropically, using a solution of $35 \mathrm{wt} . \% \mathrm{KOH}$ in water (Fig. 1c), ruled by the following reaction [39]:

$$
\mathrm{Si}+2 \mathrm{OH}^{-}+2 \mathrm{H}_{2} \mathrm{O} \rightarrow \mathrm{SiO}_{2}(\mathrm{OH})_{2}^{2-}+2 \mathrm{H}_{2} \text {. }
$$

A custom-made cell was used in this step to selectively remove the substrate on an area of $1 \mathrm{~mm}^{2}$. The process was carried out at a controlled temperature of $80^{\circ} \mathrm{C}$ inside a furnace Selecta 210 (Grupo Selecta, Abrera, Spain). The typical etch rate for these conditions was $50 \mu \mathrm{m} / \mathrm{h}$. The purpose of wet etching was not only patterning the substrate but also minimizing its thickness, since this optical layer adds noise to the PSi transmittance spectra as well as absorbs part of it. Ideally, the resulting thickness equals the size of the PSi structure. However, besides the difficulty entailed, extremely low thicknesses lead to a fragility increase. For that reason, we reduce the Si substrate thickness only down to $20 \mu \mathrm{m}$, which took approximately 7 
hours. A profiler Dektak 150 (Veeco, Plainview, USA) was used for measuring the resulting thickness as well as the width of the windows. Afterwards, each piece was dipped in a $48 \mathrm{wt}$. $\%$ solution of $\mathrm{HF}$ in $\mathrm{H}_{2} \mathrm{O}$ for $30 \mathrm{~s}$ to remove the $\mathrm{SiO}_{2}$ mask layer.

For the preparation of PSi, the electrochemical anodization of the $\mathrm{Si}$ substrate was performed under galvanostatic conditions with a custom-made Polytetrafluoroethylene (PTFE) cell using an electrolyte that contained $\mathrm{HF}$ and EtOH in a 1:2 volumetric ratio. The chemical reactions were well described by Föll et al. [9]. A platinum electrode immersed in the electrolyte worked as anode and a copper film in contact with the back side of the substrate worked as cathode. The anodization current was supplied by an Agilent E3648A power generator (Santa Clara, USA), remotely controlled with a LabVIEW-based application (National Instruments, Austin, USA). We anodized the wet etched side of the sample to achieve a better uniformity of the structures. This also restricted the porosification to the patterned areas. Anodizing the back side of the substrate would have resulted in porosified regions unsuitable for sensing, that would have been unnecessarily filled with the analyzed substances, reducing the available net volume.

A monolayer structure was formed using $46 \mathrm{~mA} / \mathrm{cm}^{2}$ for 500 $\mathrm{s}$ (Fig. 1d). We chose this structure because closely-spaced fringes are formed in the NIR region, where the equipment used during the experiments recorded the spectra. The position of each fringe is given by the next equation [40], where $\lambda$ is the wavelength position, $n$ the RI, $h$ the thickness and $m$ an integer sorting all the fringes:

$$
\lambda_{m}=2 h n / m \text {. }
$$

Then, in order to open the end of the pores, the backside of the substrate was electropolished with a current density of 450 $\mathrm{mA} / \mathrm{cm}^{2}$ for $60 \mathrm{~s}$. By doing this, we created suspended PSi membranes with open-ended pores at both ends, as demonstrated by Field Emission Scanning Electron Microscopy (FESEM) images taken at both sides of the structure (Fig. 2). The topography was studied using the ImageJ processing

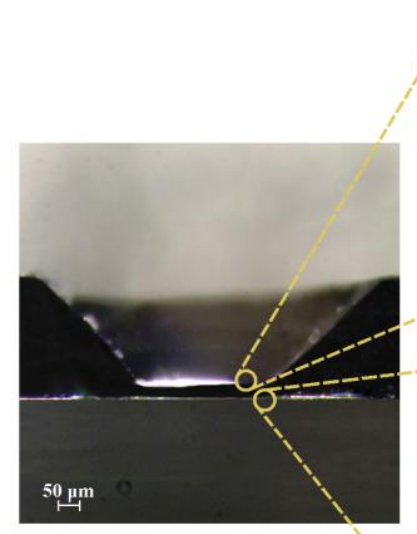

(a)

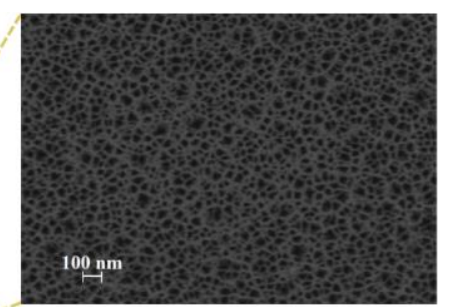

(b)

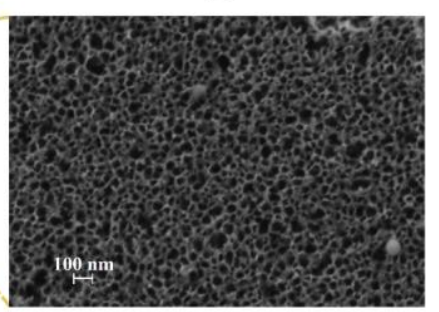

(c)
Fig. 2. Physical characterization of the suspended PSi membranes. (a) Optical microscope image of substrate section showing the reduced-thickness area obtained after wet etching; (b) Top side of PSi surface (wet etched side); (c) Bottom side of PSi surface (electropolished side).

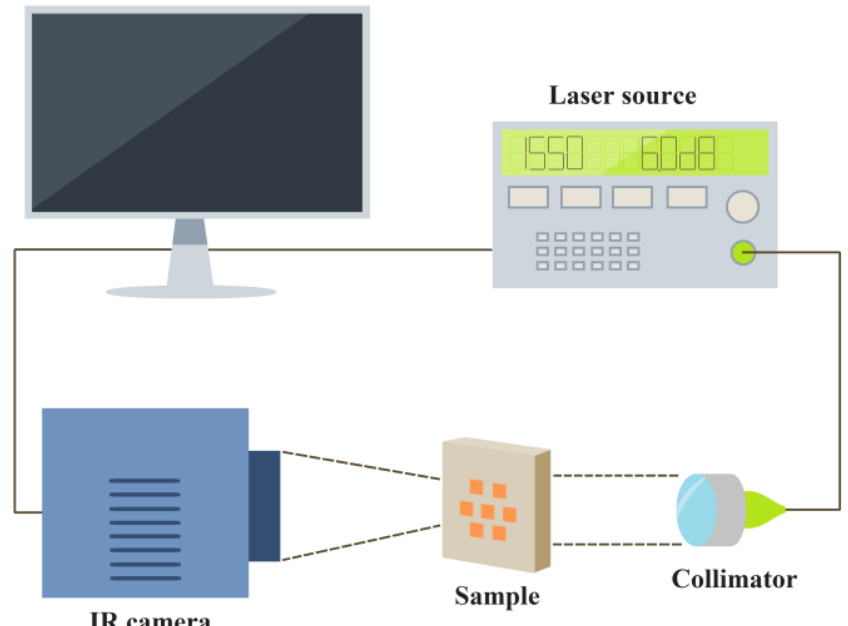

Fig. 3. Horizontal optical setup employed for simultaneous RI sensing using an array of suspended PSi membranes.

software [41] and consistency in pore density and pore diameter values at both ends of the structure proved a good vertical uniformity. More information about the characterization of these kind of structures can be found in our previous work [42].

Then, the reflectance spectra of the samples were characterized using a Fourier-Transform Infrared (FTIR) spectroscopy system (Bruker, Billerica, USA). The measurements were performed in the near-infrared range (NIR) from 1100 to $2500 \mathrm{~nm}$. The spectrometer employed a liquid $\mathrm{N}_{2}$ cooled MTC326 detector. All spectra were recorded at $4 \mathrm{~cm}^{-1}$ resolution and averaged using 32 scans.

A simulation program based on the Transfer Matrix Method (TMM) [43] was developed to model the transmittance spectra of an arbitrary number of layers and used both for designing the porous structure and fitting the FTIR measurements in order to characterized parameters such as the effective RI and the thickness.

\section{B. Optical system}

Removing the substrate under the porous structure was performed both for patterning the sensor and, at the same time, for improving the transmittance spectra of the suspended PSi membranes. Measuring the transmittance instead of the reflectance of the sensor reduced the complexity of our sensing platform and expedited the alignment time.

As a source, we used an 81980A compact tunable laser of Keysight Technologies (Santa Rosa, USA) operating in the range from 1465 to $1575 \mathrm{~nm}$, with a wavelength resolution of $20 \mathrm{pm}$ and an output power of $6 \mathrm{dBm}$. We used Eq. 2 to design the spectral shape of the suspended PSi membranes so that at least two fringes were found in this wavelength range, which were later used for tracking purposes during the sensing experiments. The output was connected to a collimator F230FC-1550 (Thorlabs, Newton, USA) with 0.53 numerical aperture and $4.67 \mathrm{~mm}$ field number. With this configuration, we achieved a highly directional beam of approximately $7 \mathrm{~mm}^{2}$.

As a recording device, we used an infrared camera XEVA320 of Xenics (Heverlee, Belgium), based on an InGaAs detector, focused with an Olympus PLN 20x objective 


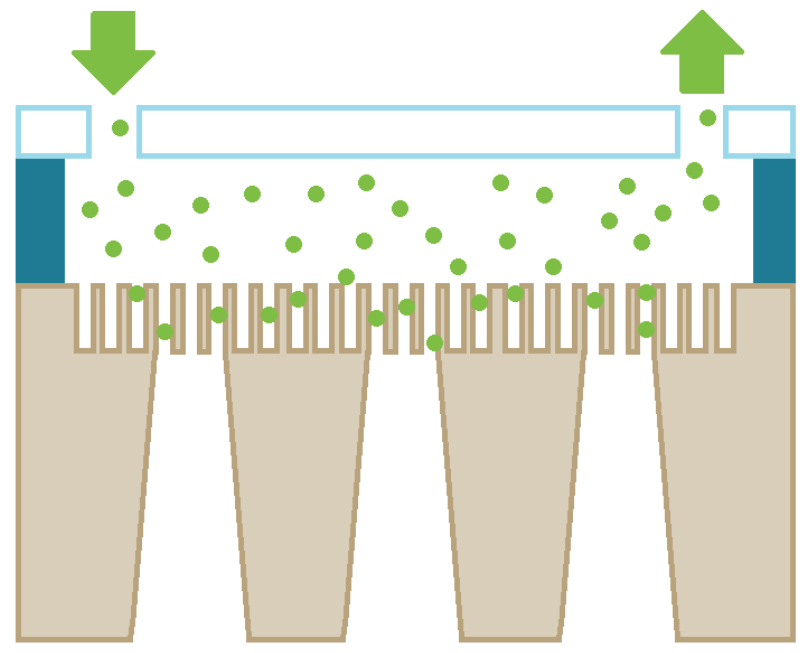

Notes:

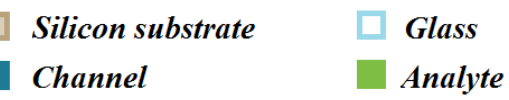

Fig. 4. Schematic illustration of the fluidic cell consisting of a channel and a glass slide to create a sealed path for the liquids substances to flow over the PSi membranes.

(Shinjuku, Japan), 0.4 numerical aperture and $22 \mathrm{~mm}$ field number, and placed close to the backside of the sensor. This camera offers high frame rates, which makes it suitable for realtime applications, with a 320x256 pixel resolution.

Both elements, the source and the camera, were positioned facing each other and precisely aligned to maximize the measured intensity. The array of sensors was placed between them and mounted on a 3-axis stage that allowed a fast alignment of the sample in a few seconds (Fig. 3). We focused a total area of $1.25 \times 1.25 \mathrm{~mm}^{2}$, which means that each pixel represented $20 \mu \mathrm{m}^{2}$. The sensor was integrated in a fluidic cell in order to flow liquid substances over the pores. This cell consisted of a $250 \mu \mathrm{m}$ thick microfluidic channel sealed with a glass slide of $1 \mathrm{~mm}$ of thickness, where an inlet and an outlet were built to connect the tubes that led the substances from the sample container to the withdrawing pump, respectively (Fig. 4). Another reason to measure the transmittance spectrum instead of the reflectance was avoiding a second trip of the optical signal through the fluidic cell, which would have

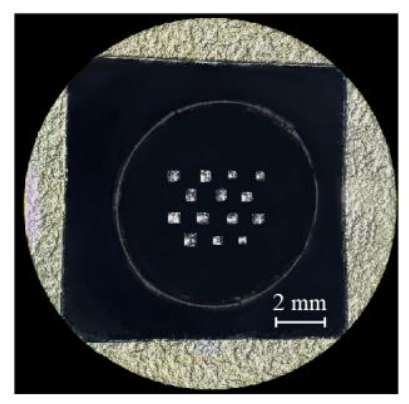

(a)

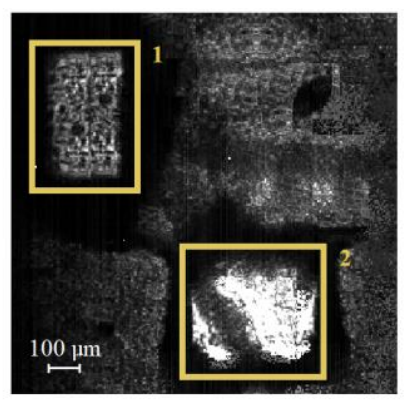

(b)
Fig. 5. (a) Microscope image of the Si substrate after wet etching the 2D matrix pattern; (b) Infrared photography of a fabricated array of sensors using the XEVA-320 infrared camera. The regions of interest selected using the LabVIEW-based program correspond each one with a PSi sensing spot. involved a higher attenuation and, hence, a decrease of the noise and a limitation of the LoD.

The optical system was remotely controlled by an application based on LabVIEW, that swept the wavelength of the laser in synchronization with the image acquisition of the camera, at a rate of $20 \mathrm{~nm} / \mathrm{s}$ and a resolution of $20 \mathrm{pm}$. The transmittance spectrum at each pixel was composed as the intensity variation within the wavelength range. An initial configuration of the program allowed us to select automatically or manually regions of interest (ROI) to analyze separately each sensing spot (Fig. 5). The average spectrum of all the pixels within those ROI was calculated, and the evolution of a spectral feature tracked through time.

We realized a series of experiments flowing substances with different RI to characterize the performance of the array of suspended PSi membranes. We used DIW, EtOH and IPA, having each a refractive index of $1.3325,1.3505$ and 1.3673 RIU respectively, at $1550 \mathrm{~nm}$. We flowed the substances in cycles of $5 \mathrm{~min}$ at a rate of $20 \mu \mathrm{L} / \mathrm{min}$. The infiltration of those liquids into the pores caused the variation of the effective RI of the porous layer, described by the effective medium approximation of Looyenga for highly porous materials [15]:

$$
n=\left((1-P) n_{s}^{2 / 3}+P n_{m}^{2 / 3}\right)^{3 / 2}
$$

where $n$ is the effective RI of the porous layer, $n_{s}$ is the substrate RI, $n_{m}$ the RI of the medium filling the pores and $P$ the porosity. As it can be deducted from Eq. 2, an increase of $n$ will increase the resonance wavelength, which is seen as a shift of the spectrum. Recording the transmittance while flowing all the different substances and tracking the displacement of a fringe allowed us to measure when the RI change took place and quantify it.

Additionally, after the experiments, all recorded data was processed using a program based on Matlab (The Mathworks, Natick, USA) to filter out the high frequency noise and compute the sensitivity, S, of each sensing spot, defined as wavelength shift over the RI variation of the medium:

$$
S=\Delta \lambda / \Delta n \text {. }
$$

We also used this program to characterize the full width at half maximum (FWHM) of the fringes, the free spectral range (FSR), the Q-factor, the noise and the figure of merit (FOM).

\section{RESULTS}

\section{A. Suspended PSi membranes}

A series of samples were fabricated following the four-step process described above. Fig. 6 shows one profile after wet etching. We were able to achieve thicknesses as thin as $20 \mu \mathrm{m}$, with a roughness of only $50 \mathrm{~nm}$ and a $5 \%$ variation among all the exposed areas, approximately. Beforehand, we had studied the etching conditions to minimize the roughness, in order to reduce the dispersion of the transmitted light. As depicted in Fig. 6, due to the selectivity of $\mathrm{KOH}$ etching, the $\mathrm{SiO}_{2}$ mask layer withstood the whole process long enough to protect the unexposed substrate, being only reduced a few hundred of nanometers. We measured a dissolution rate of approximately $0.1 \mathrm{~nm} / \mathrm{s}$ for this material, under our etching conditions. The 


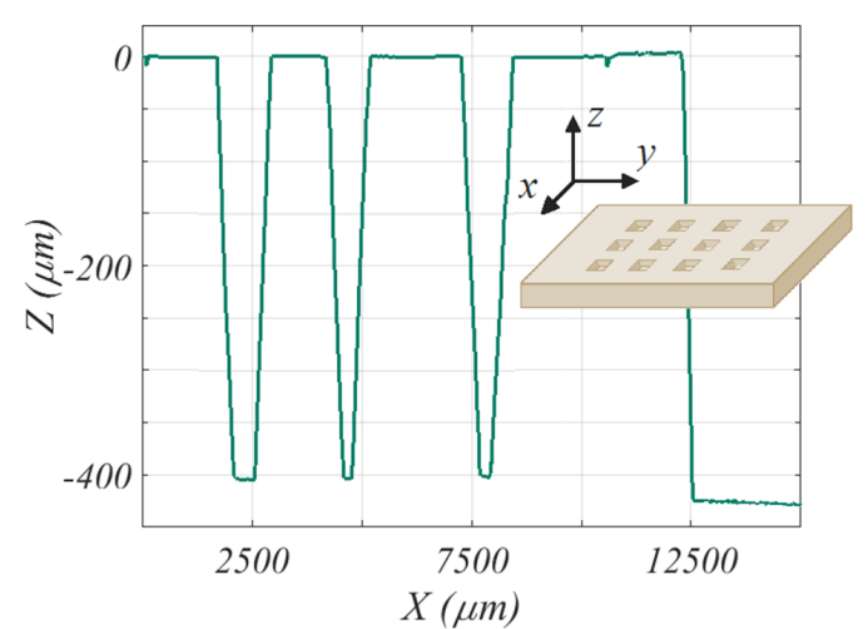

Fig. 6. Profile of a $425 \pm 5 \mu \mathrm{m}$ thick piece of $\mathrm{Si}$ wafer with three reducedthickness regions. A wet etching of the substrate caused the dissolution of approximately $405 \mu \mathrm{m}$ in the $\mathrm{Z}$ axis and variable widths between 150 and 500 $\mu \mathrm{m}$ in the $\mathrm{X}$ axis. Notice that a $3.55 \pm 0.05 \mu \mathrm{m}$ thick silicon dioxide mask layer was deposited on top of the Si substrate and only a few hundreds of nanometers remained after the wet etching.

reproducibility of this process was good if the etching parameters were maintained, with a variation below $10 \%$.

We tested our method using patterns of lines and squares and were able to create features as small as $150 \times 150 \mu \mathrm{m}^{2}$, with a pitch of $600 \mu \mathrm{m}$. This means that the maximum density of transducers that could have been integrated in the sample was 300 sensing spots $/ \mathrm{cm}^{2}$. Yet, using a $\mathrm{Si}$ wafer with an initial thickness of $150 \mu \mathrm{m}$ could have tripled this value.

After anodizing the samples, we obtained suspended PSi membranes with an effective RI of $1.4 \pm 0.1$ measured at 1550 $\mathrm{nm}$. According to Eq. 3, that corresponded to a porosity of $77 \pm$ $2 \%$. The total thickness of the structure was $20 \pm 0.5 \mu \mathrm{m}$ and the average pore diameter was $15 \pm 5 \mathrm{~nm}$. Multiple fringes were formed closely spaced in the NIR (Fig. 7), with a Q-factor of approximately 60 at $1550 \mathrm{~nm}$. The theoretical Q-factor that we estimated for that structure using the TMM-based program had

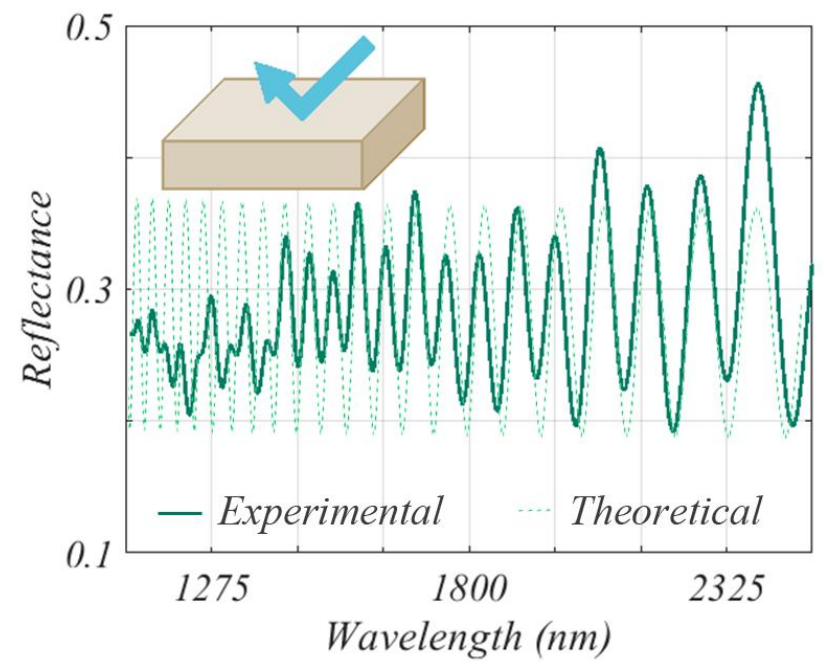

Fig. 7. FTIR reflectance spectra (solid line) of the fabricated structure between 1100 and $2500 \mathrm{~nm}$, measured on the top side of the PSi monolayer, and theoretical reflectance spectra (dotted line) calculated using a simulation program based on the Transfer Matrix Method (TMM).

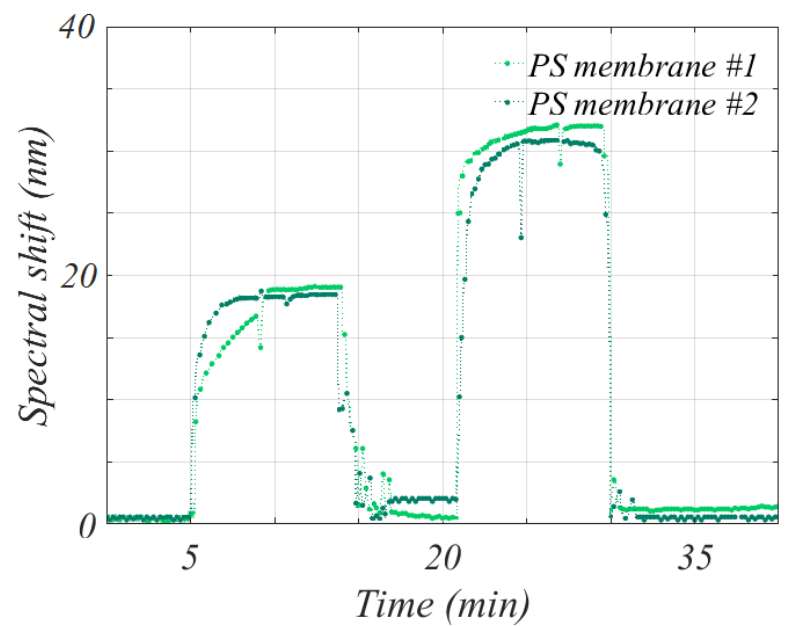

Fig. 8. Normalized spectral shift of the transmittance of two PSi membranes while flowing DIW from the start of the experiment to $t=5 \mathrm{~min}$, IPA from $\mathrm{t}=5$ $\min$ to $t=14 \min$, DIW from $t=14 \min$ to $t=21 \mathrm{~min}, \mathrm{EtOH}$ from $t=21 \mathrm{~min}$ to 29 $\min$ and DIW from $t=29$ until the end of the experiment.

been 75 at $1550 \mathrm{~nm}$. This difference is attributed to the vertical non-uniformity of the pore size. No additional passivation was performed to stabilize the optical properties of the PSi and improve its shelf-life [44].

\section{B. Simultaneous RI sensing}

We demonstrated the feasibility of the array of $\mathrm{PSi}$ transducers performing a series of RI sensing experiments. Fig. 8 shows the simultaneous spectral tracking of two independent PSi membranes, as a proof of concept of our work. We used DIW as a baseline and measured the spectral shifts respect to this one. When IPA was flowed, we calculated a displacement of $35.5 \pm 0.8 \mathrm{~nm}$ and $35.6 \pm 0.6 \mathrm{~nm}$ for each suspended PSi membrane. In the same way, when EtOH was flowed, we measured a shift of $19.2 \pm 0.7 \mathrm{~nm}$ and $19.7 \pm 0.7 \mathrm{~nm}$. We believe that the mismatch between both sensing spots was the result of slight structural and spectral differences caused by the anodization of Si. Nevertheless, both membranes displayed a very similar behavior both in displacement and in time. The arrival of a new substance triggered a rapid shift of the spectrum towards longer or shorter wavelengths, whether it was an increase or decrease of the RI of the medium filling the pores. Typically, between 3 and 4 min elapsed before the spectrum became stable in the new position. That was the time required to replace the medium formerly filling the pores with the new one. Since this replacement was realized by diffusion, properties such us density, viscosity and hydrophobicity may affect the transition period.

Sensitivity was calculated as a linear regression of the spectral shift for each RI variation (Fig. 9). For this purpose, we used data from several experiments where we measured the displacements caused by DIW, 20wt\% EtOH in DIW, EtOH and IPA. As a result, we obtained an experimental S of $680 \pm$ $15 \mathrm{~nm} / \mathrm{RIU}$ and $690 \pm 15 \mathrm{~nm} / \mathrm{RIU}$ respectively for each PSi membrane, and a FOM, defined as S/FWHM, of $27.2 \mathrm{RIU}^{-1}$ and $27.6 \mathrm{RIU}^{-1}$ respectively. The model based on the TMM was employed to calculate the theoretical values for this porous layer. A maximum theoretical sensitivity of $900 \mathrm{~nm} / \mathrm{RIU}$ was estimated for the fabricated structures at a wavelength of 1550 


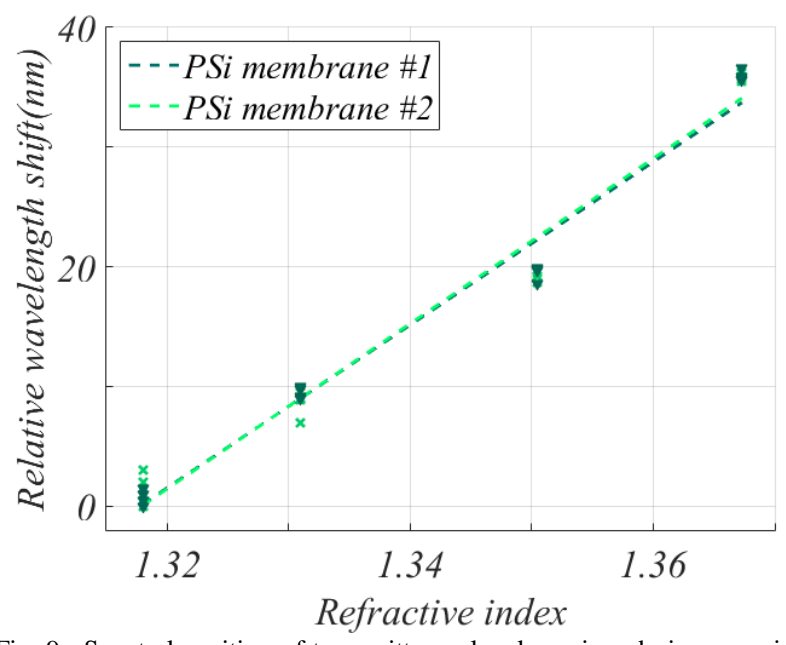

Fig. 9. Spectral position of transmittance local maxima during experimental measurements for every flowed substance, i.e. DIW, 20\% w/w EtOH, EtOH and IPA whose RI at $1550 \mathrm{~nm}$ were $1.318,1.331,1.3505$ and 1.3673 respectively. Two groups are displayed in the graph, corresponding each one with a suspended PSi membrane, where marks represent measurements and lines are their linear regressions.

$\mathrm{nm}$, with a FOM equal to $44 \mathrm{RIU}^{-1}$. The averaged experimental sensitivity of the sensor was $685 \mathrm{~nm} / \mathrm{RIU}$, which represents $75 \%$ of the estimated theoretical value.

As we demonstrated in a previous work [10], this decrease is caused by the nanostructure of porous materials, which hinders the infiltration of the samples. Small pore diameter, wall roughness and tortuosity increase the resistance of penetration into the pores, which causes bad diffusion of the substances and air entrapment. Moreover, PSi is hydrophobic [45] and waterbased solutions might not be able to enter the pores at all. According to our previous work, the sensitivity decrease was in direct proportion to the volume filled by the substance and, thus, it can be concluded that a $75 \%$ decrease is equivalent to say that $25 \%$ the structure was not filled. Oxidizing the sensor could improve the infiltration yet not maximize it but, due to the expanded volume of oxide, only structures with pore sizes above $30 \mathrm{~nm}$ can benefit from that. A good solution would be implementing an active flow where the substances were forced to flow through the pores. As we proved in another work [46], it is possible to reach the theoretical sensitivity experimentally by doing this. Unfortunately, we were not able to implement the active flow in this study due to some limitations such as the complexity of the fluidic cell, the attenuation of the optical signal and the flow resistance of the porous layer, some of which could be overcome increasing the pore size, reducing more the thickness of the substrate and fabricating structures with higher Q-factor.

We detected RI variations down to $10^{-2}$ RIU. Using the baseline of the experiments, we measured a noise of $50 \mathrm{pm}$ (standard deviation), which means that the theoretical LoD of our system was $10^{-5} \mathrm{RIU}$. This value is higher than the state-ofthe-art but is enough for some applications [47]. We were able to obtain that limit of detection with such a simple optical platform by reducing the window where we measured the transmittance spectra. This allowed us to improve the resolution and reduce the noise. Decreasing the wavelength range also expedited the acquisition rate. We recorded the signal at a rate of $0.08 \mathrm{~Hz}$. Since the transition time, where the medium filling the pores is changing, is above $3 \mathrm{~min}$, with that rate we acquired enough samples to consider that the sensing response was on real-time. As it has been discussed, the response time of the sensor is limited by the diffusion of the substances into the pores. Flowing through the pores can reduce this transition since the substances are forced to infiltrate the pores and there is no mixture between different mediums.

The capabilities of this platform in the field of multiplexed detection are very promising. If we used masks with a pattern of $150 \times 150 \mu \mathrm{m}^{2}$ features, we could potentially obtain a membrane size such that each pixel of the IR camera would represent a single sensing spot, which means that this system could potentially handle up to 4000 sensing spots $/ \mathrm{cm}^{2}$ simultaneously and on real-time.

\section{CONCLUSION}

We have developed a fast, low-cost and easy-to-implement four-step microfabrication process to obtain an array of rectangular-shaped suspended PSi membranes with tunable optical properties and a potential density of 1000 sensing spots $/ \mathrm{cm}^{2}$, suitable to be used as a substrate for the fabrication of other photonic structures and compatible with chemical patterning. One of the key aspects of our approach was the combination of well-known processes such as wet etching using $\mathrm{KOH}$, the oxidation of $\mathrm{Si}$ and the formation of PSi in a particular sequence that allowed us to reduce the number of steps, without limiting the extent of each technique by itself, and being able to create an interesting structure such as the suspended membranes, thin layers of porous material with a high sensitivity to the RI variation of the medium filling the pores and with an increased robustness due to the attachment to the substrate by the edges.

We demonstrated the feasibility of our sensor based on multiple PSi sensing spots performing simultaneous RI sensing experiments. Our optical system had a user-friendly configuration with very high capabilities but low specification requirements, easily-scalable to handle multiplexed detection and able to give a real-time response. We proposed the measurement of the transmittance spectrum instead of the reflectance as a way to facilitate the alignment of the sensors and reduce the attenuation of the signal. We calculated an experimental sensitivity of $685 \mathrm{~nm} / \mathrm{RIU}$, which is close to the order of magnitude of the state-of-the-art using a low-cost sensor.

\section{REFERENCES}

[1] C.I.L. Justino, A.C. Duarte, T.A.P. Rocha-Santos, "Recent progress in biosensors for environmental monitoring: a review," Sensors, vol. 17, no. 2918, 2017.

[2] O. Lazcka, F.J. Del Campo, F.X. Muñoz, "Pathogen detection: a perspective of traditional methods and biosensors," Biosens. Bioelectron., vol. 22, pp. 1205-1217, 2007.

[3] B.T. Cunningham, "Label-free optical biosensors: an introduction,." in Label-free biosensors: techniques and applications, 1st ed., M.A. Cooper, Ed., Cambridge University Press: Cambridge, United Kingdom, 2009, pp. 128. 
[4] J. Hopkins, K. Fidanovski, A. Lauto, D. Mawad, "Allorganic semiconductors for electrochemical biosensors: an overview of recent progress in material design," Front. Bioeng. Biotechnol., vol. 7, no. 237, 2019.

[5] J. Peña-Bahamonde, H.N. Nguyen, S.K. Fanourakis, D.F. Rodrigues, "Recent advances in graphene-based biosensor technology with applications in life sciences," J. Nanobiotechnol., vol. 16, no. 75, 2018.

[6] N. Tripathy, D. Kim, "Metal oxide modified $\mathrm{ZnO}$ nanomaterials for biosensor applications," Nano Convergence, vol 5, no. 27, 2018.

[7] L. Ge, Q. Liu, N. Hao, W. Kun, "Recent developments of photoelectrochemical biosensors for food analysis," J. Mater. Chem. B, vol. 7, pp. 7283-7300, 2019.

[8] S. Arshavsky-Graham, N. Masaad-Ivanir, E. Segal, S. Weiss, "Porous silicon-based photonic biosensors: current status and emerging applications," Anal. Chem., vol. 91, pp. 441-467, 2019.

[9] H. Föll, M. Christophersen, J. Carstensen, G. Hasse, "Formation and application of porous silicon," Mater. Sci. Eng. R., vol. 39, no. 93, 2002

[10] R. Caroselli, D. Martín-Sánchez, S. Ponce-Alcántara, F. Prats-Quílez, L. Torrijos-Morán, J. García-Rupérez, "Realtime and in-flow sensing using a high sensitivity porous silicon microcavity-based sensor," Sensors, vol. 17, no. 2813, 2017.

[11] I. Rea, A. Lamberti, I. Rendina, G. Coppola, M. Gioffrè, M. Iodice, M. Casalino, E. De Tommasi, L. De Stefano, "Fabrication and characterization of a porous silicon based microarray for label-free optical monitoring of biomolecular interactions," J. Appl. Phys., vol. 107, no. 014513, 2010.

[12] S. Seo, H.R. Azmand, A.N. Enemuo, "Hollow core waveguide sensor array based on a microporous silicon membrane structure," J. Light. Technol., vol. 37, no. 9, pp. 2036-2041, 2019.

[13] S. Surdo, F. Carpignano, S. Merlo, G. Barillaro, "Nearinfrared silicon photonic crystals with high-order photonic bandgaps for high-sensitivity chemical analysis of waterethanol mixtures," ACS Sens., vol. 3, pp. 2223-2231, 2018.

[14] D.A.G. Bruggeman, "Berechnung verschiedener physikalischer konstanten von heterogenen substanzen, dielektrizitätskonstanten und leitfähigkeiten der mischkörper aus isotropen substanzen," Ann. Phys., vol. 24, pp. 636-664, 1935.

[15] H. Looyenga, "Dielectric constants of heterogeneous mixtures," Physica, vol. 31, pp. 401-406, 1965.

[16] S. Sahu, J. Ali, P.P. Yupapin, G. Singh, "Porous silicon based Bragg-grating resonator for refractive index biosensor," Photonic Sens., vol. 8, no. 3, pp. 248-254, 2018.

[17] S. Mariani, L.M. Strambini, A. Paghi, G. Barillaro, "Lowconcentration ethanol vapor sensing with nanostructured porous silicon interferometers using interferogram average over wavelength reflectance spectroscopy," IEEE Sens. J., vol. 18, no. 19, pp. 7842-7849, 2018.

[18] G.A. Rodriguez, P. Markov, A.P. Cartwright, M.H. Choudhury, F.O. Afzal, T. Cao, S.I. Halimi, S.T. Retterer, I.I. Kravchenko, S.M. Weiss, "Photonic crystal nanobeam biosensors based on porous silicon," Opt. Express, vol. 27, no. 7, 9536, 2019.

[19] D. Basu, T. Sarkar, K. Sen, S.M. Hossain, J. Das, "Multiparametric optical glucose sensor based on surface functionalized nano-porous silicon,” IEEE Sens. J., vol. 18, no. 24, pp. 9940-9947, 2018.

[20] M. Vakili, M. Noori, "A highly accurate refractive index sensor with two operation modes based on photonic crystal ring resonator," Ann. Phys., vol. 531, no. 1800453, 2019.

[21] A.M. Ahmed, A. Mehaney, "Ultra-high sensitive 1D porous silicon photonic crystal sensor based on the coupling of Tamm/Fano resonances in the mid-infrared region," Sci. Rep., vol. 9, no. 6973, 2019.

[22] I. Petrova, V. Konopsky, I. Nabiev, A. Sukhanova, "Labelfree flow multiplex biosensing via photonic crystal surface mode detection," Sci. Rep., vol. 9, no. 8745, 2019.

[23] D. Semenova, Y.E. Silina, M. Koch, L. Micheli, A. Zukov, K.V. Gernaey, "Sensors for biosensors: a novel tandem monitoring in a droplet towards efficient screening of robust design and optimal operating conditions," Analyst, vol. 144, pp. 2511-2522, 2019.

[24] T. Ayupova, M. Shaimerdenova, S. Korganbayev, M. Sypabekova, A. Bekmurzayeva, W. Blanc, S. Sales, T. Guo, C. Molardi, D. Tosi, "Fiber optic refractive index distributed multi-sensors by scattering-level multiplexing with $\mathrm{MgO}$ nanoparticle-doped fibers," IEEE Sens. J., early access, 2019.

[25] I.S. Kucherenko, O.O. Soldatkin, Y.V. Topolnikova, S.V. Dzyadevych, A.P. Soldatkin, "Novel multiplexed biosensor system for the determination of lactate and pyruvate in blood serum," Electroanalysis, vol. 31, pp. 1608-1614, 2019.

[26] R. Hu, X. Zhang, Q. Xu, D. Lu, Y. Yang, Q. Xu, Q. Ruan, L. Mo, X. Zhang, "A universal aptameric biosensor: multiplexed detection of small analytes via aggregated perylene-based broad-spectrum quencher," Biosens. Bioelectron., vol. 92, pp. 40-46, 2017.

[27] B.S. Flavel, M.J. Sweetman, C.J. Shearer, J.G. Shapter, N.H. Voelcker, "Micropatterned arrays of porous silicon: toward sensory biointerfaces," ACS Appl. Mater. Interfaces, vol. 3, pp. 2463-2471, 2011.

[28] Y. Zhu, A.H. Soeriyadi, S.G. Parker, P.J. Reece, J.J. Gooding, "Chemical patterning on preformed porous silicon photonic crystals: towards multiplex detection of protease activity at precise positions," J. Mater. Chem. B, vol. 2, no. 3582, 2014.

[29] F.S.H. Krismastuti, A. Cavallaro, B. Prieto-Simon, N.H. Voelcker, "Toward multiplexing detection of wound healing biomarkers on porous silicon resonant microcavities," Adv. Sci., vol. 3, no. 1500383, 2016.

[30] R. Liu, W. Li, T. Cai, Y. Deng, Z. Ding, Y. Liu, X. Zhu, X. Wang, J. Liu, B. Liang, T. Zheng, J. Li, "TiO2 nanolayerenhanced fluorescence for simultaneous multiplex mycotoxin detection by aptamer microarrays on a porous silicon surface," ACS Appl. Mater. Interfaces, vol. 10, pp. 14447-14453, 2018.

[31] S. Borini, A.M. Rossi, L. Boarino, G. Amato, "Patterning of porous silicon by electron-beam lithography," J. Electrochem. Soc., vol. 150, pp. G311-G313, 2003.

[32] T. Deng, Y. Wang, Q. Chen, H. Chen, Z. Liu, "Massive fabrication of silicon nanopore arrays with tunable shapes," Appl. Surf. Sci. 2016, 390, 681-688, 2016.

[33] L. Chen, Z. Chen, J. Wang, S. Xiao, Z. Lu, Z. Gu, L. Kang, J. Chen, P. Wu, P. Tang, J. Liu, "Gel-pad microarrays templated by patterned porous silicon for dual-mode detection of proteins," Lab Chip, vol. 9, pp. 756-760, 2009.

[34] J. Pla-Tolós, Y. Moliner-Martínez, C. Molins-Legua, P. Campíns-Falcó, "Solid glucose biosensor integrated in a multi-well microplate coupled to a camera-based detector: application to the multiple analysis of human serum samples," Sensor Actuat. B-Chem., vol. 258, pp. 331-341, 2018.

[35] S.B. Patil, D.S. Dheeman, M.A. Al-Rawhani, S. Velugotla, B. Nagy, B.C. Cheah, J.P. Grant, C. Accarino, M.P. Barret, D.R.S. Cumming, "An integrated portable system for single chip simultaneous measurement of multiple disease associated metabolites," Biosens. Bioelectron., vol. 122, pp. 88-94, 2018.

[36] M. Iqbal, M.A. Gleeson, B. Spaugh, F. Tybor, W.G. Gunn, M. Hochberg, T. Baehr-Jones, R.C. Bailey, L.C. Gunn, "Label-free biosensor arrays based on silicon ring resonators and high-speed optical scanning instrumentation," IEEE J. Sel. Top. Quantum Electron., vol. 16, no. 3, pp. 654-661, 2010.

[37] F. Yesilkoy, "Optical interrogation techniques for nanophotonic biochemical sensors," Sensors, vol. 19, no. 19, 2019.

[38] Y. Zhou, J. Wu, Z. Bian, J. Suo, G. Zheng, Q. Dai, "Fourier ptychographic microscopy using wavelength multiplexing," J. Biomed. Opt., vol. 22, no. 6, 2017.

[39] R.A. Wind, M.A. Hines, "Macroscopic etch anisotropies and microscopic reaction mechanisms: a micromachined structure for the rapid assay of etchant anisotropy," Surf. Science, vol. 460, pp. 21-38, 2000. 
[40] S. Ponce-Alcántara, D. Martín-Sánchez, M. Kovylina, A. Pérez-Márquez, J. Maudes, N. Murillo, J. García-Rupérez, "Dual refractive index and viscosity sensing using polymeric nanofibers optical structures," IEEE Sens. J., vol. 19, no. 24, pp. 11850-11857, 2019.

[41] W. S. J. Rasband, "ImageJ," U.S. Nat. Inst. Health, Bethesda, MD, USA,Tech. Rep. 1.51n, 2016. [Online]. Available: https://imagej.nih.gov/ij/.

[42] D. Martín-Sánchez, S. Ponce-Alcántara, P. Martínez-Pérez, J. García-Rupérez, "Macropore formation and pore morphology characterization of heavily doped p-type porous silicon," J. Electrochem. Soc., vol. 166, no. 2, pp. B9-B12, 2019.

[43] R.B. Balili, “Transfer Matrix Method in nanophotonics," Int. J. Mod. Phys., vol. 17, pp. 159-168, 2012.

[44] J. Rasson, L.A. Francis, "Improved stability of porous silicon in aqueous media via atomic layer deposition of oxides," $J$. Phys. Chem. C, vol 122, pp. 331-338, 2018.

[45] D.B. Mawhinney, J.A.Glass, J.T.Yates, "FTIR study of the oxidation of porous silicon,"J. Phys. Chem. B, vol. 101, no. $1202,1997$.

[46] D. Martín-Sánchez, S. Ponce-Alcántara, J. García-Rupérez, "Sensitivity comparison of a self-standing porous silicon membrane under flow-through and flow-over conditions," IEEE Sensors J., vol. 19, pp. 3276-3281, 2019.

[47] M. Olivia-Ramírez, J. Gil-Rostra, F. Yubero, A.R. GonzálezElipe, "Robust polarization active nanostructured 1D Bragg Microcavities as optofluidic label-free refractive index sensor," Sens. Actuat. B-Chem., vol. 256, pp. 590-599, 2018.

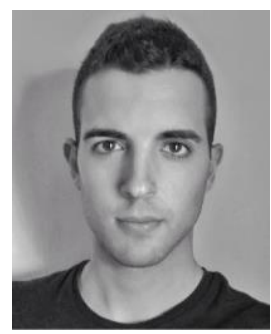

David Martín-Sánchez received the bachelor degree in Telecommunications Engineering from the University of Seville, Spain, in 2014, and the master degree in Biomedical Engineering from the Universitat Politècnica de València, Spain, in 2016. Since 2016, he has been a Research Assistant with the Biophotonics Research Group, in the Nanophotonics Technology Center of Valencia (NTC). In 2019, he was awarded with the Ph.D. in Telecommunication. His research interests include the design, fabrication, and characterization of photonic sensors based on porous silicon.

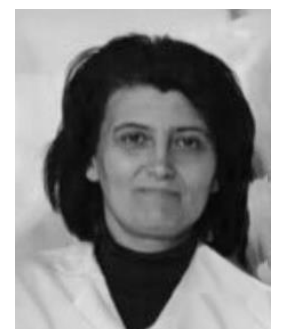

Todora I. Angelova received her M.S. degree in physics in 1998, from Sofia's University "St. Kliment Ohridsky", Bulgaria. She received her Ph.D. degree in 2010, at the Applied Physic Department of the University of Valencia, Spain. Currently, she is a Process Development Engineer at the Nanophotonics Technology Center of Valencia (NTC). Her research interests include the fabrication, characterization and application of graphene and thin film semiconductors materials and devices. In particular, she is active in experimental studies on Plasma Enhanced Chemical Vapour Deposition (PECVD) layers. Her work can also be filled with knowledge in characterization techniques like Raman scattering, Spectroscopic Ellipsometry and Atomic force microscopy (AFM).

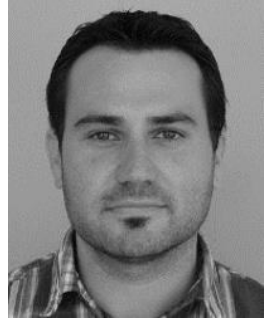

Jaime García-Rupérez (M'03-SM'16) received the M.S. and Ph.D. degrees in telecommunication from the Universitat Politécnica de Valéncia, Spain, in 2002 and 2008, respectively. $\mathrm{He}$ is Associate Professor in the Universitat Politècnica de València since 2005 and Leader of the Biophotonics Research Group from the Nanophotonics Technology Centre since 2008. His work focuses on the development of integrated photonic biosensing systems for its application in fields as medical diagnosis, environmental control or biological safety. Dr. García-Rupérez has authored over 140 journal and conference publications, one book chapter, and four patents. 